\title{
Pattern of antimalaria treatment and risk factors for torsades de pointes in admitted heart failure patients in Lagos-Nigeria
}

\author{
Janet N. Ajuluchukwu ${ }^{1}$, Emmanuel N. Anyika ${ }^{2}$, Christiana A. Boyle ${ }^{2}$ \\ 1. Department of Medicine, College of Medicine, University of Lagos, Lagos, Nigeria. 2. Department of Clinical Pharmacy \\ and Biopharmacy, Faculty of Pharmacy, University of Lagos, Lagos, Nigeria.
}

Correspondence: Janet N. Ajuluchukwu. Address: Department of Medicine, College of Medicine, University of Lagos, Lagos, Nigeria. E-mail: ajulujay@gmail.com

Received: April 24, 2014

Accepted: May 21, 2014

Online Published: July 4, 2014

DOI : 10.5430/jha.v3n5p161

URL: http://dx.doi.org/10.5430/jha.v3n5p161

\section{Abstract}

Background: In a malaria-holoendemic region, concurrent malaria complicating heart failure (HF) occurs; with higher morbidity and increased adverse drug-drug interactions.

Aim: To characterise malaria treatment and risk factors of cardio-toxicity among HF patients.

Objectives: To characterise the use of anti-malaria agents (AMA), compare risk factors of torsades de pointes (TdP) amongst AMA-users and non-users, and to assess length of hospital stay.

Methods: Admitted HF patients were retrospectively studied, and grouped on the basis of malaria treatment. TdP risk factors- advanced age, bradycardia, hypokalemia, and QTc prolongation were compared in the two groups.

Results: The $160 \mathrm{HF}$ patients (mean ejection fraction 39.6\% $\pm 12.6 \%$, mean age $54.9 \pm 14.6$ years) included 82 males (51.3\%), with predominant non-ischemic HF. Malaria treatment occurred in 32.5\% (52), though diagnosis was presumptive in 48 (92.3\%). All (100\%) malaria prescriptions were artemisinin-based, but monotherapeutic in $6(11.4 \%)$. Partner-drugs included sulphadoxine-pyrimethamine (SP) 26 (50\%), and lumefantrine 7 (13.5\%). TdP risk-factors of age 65 years, prolonged QTc, hypokalemia, and bradycardia occurred in 43 (26.9\%), 63 (39.4\%), 48 (30\%), and 8 $(5.0 \%)$ respectively. Group 1 (AMA treated) and group 2 patients were comparable on all mean values of risk factors. Nevertheless, affected proportions were significantly different for hypokalemia $\left(\mathrm{X}^{2}=6.1\right)$, QTc prolongation $(48.1 \%$ versus $35.2 \%, p<.05)$, and older age $(38.5 \%$ versus, $21.3 \%, p<.05 \%)$.

Conclusion: Though all HF patients similarly demonstrated risks of TdP, univariate analysis indicates a significantly higher proportion in malaria-treated patients; supporting further therapeutic caution in this patient subset.

\section{Key words}

Heart failure, Antimalaria agents, Cardiotoxicity, Torsades de Pointes-risk

\section{I ntroduction}

Heart failure (HF) is a public health issue globally, with an alarming increase in prevalence and poor prognosis despite advances in management strategies ${ }^{[1-3]}$. Heart failure has been described as a final pathway of all cardiac diseases, with a 
progressive and irreversible course ${ }^{[1,2]}$. Land-mark studies have demonstrated improved long-term survival in HF, using certain pharmacological drug classes, such as angiotensin converting enzyme inhibitors, angiotensin receptor blockers, beta blockers and aldosterone antagonists ${ }^{[1,2]}$. Thus current HF treatment guidelines recommend these drug classes in step-wise combinations, with consequent poly-pharmacy. HF patients could easily have up to five different medications on their prescriptions ${ }^{[4,5]}$. Sudden arrhythmic death may occur in HF through diverse mechanisms including ventricular tachycardia (VT), especially of the torsades de pointes (TdP) variety ${ }^{[6]}$. Some known torsadogenic risk factors include, but are not limited to female gender, hypokalemia, ECG QTc prolongation and advanced age ${ }^{[7]}$. Organic heart disease and HF are also associated with QT prolongation ${ }^{[7]}$, and such findings have been documented in HF patients in Nigeria ${ }^{[8,9]}$, and elsewhere ${ }^{[1,2]}$. Furthermore, the presence of multiple drug use; even for appropriate indications could predispose such patients to cardiotoxicity and adverse outcomes ${ }^{[7,10]}$.

In the tropics or in a malariousholoendemic area, HF patients could easily suffer concurrent malaria infection, with further need for additional malaria treatment. Currently, the treatment strategy of uncomplicated malaria in Africa is artemisininbased treatment with a combination of anti-malaria agents. Such clinical situations are thus fraught with multiple though required medications or poly-pharmacy; which further expose such patients to complex drug regimens and increased risk of cardiotoxicity ${ }^{[5,7,10]}$. Concerning HF patients, the important malaria drug adverse effects will include further cardiac depressant effects of drugs like mefloquine ${ }^{[11]}$, hypotension with 4 -aminoquinolines ${ }^{[12]}$, conduction defects with quinine, and quinidine ${ }^{[10,13]}$, or overt arrhythmias with halofantrine; which may result in sudden cardiac death ${ }^{[12-14]}$. A "pharmacologically volatile" clinical situation arises as malaria continues to be an acute health menace in high transmission areas; and prevalence of HF continues to increase ${ }^{[1,2]}$. These potential interactions are a real clinical concern. Although these two conditions are common in our community ${ }^{[5,8,9]}$, the challenges of such combined antimalaria treatment in HF patients have so far received little research attention.

Thus, the purpose of this study was to investigate the cardio-safety profile of admitted HF patients and the pattern and treatment of malaria in a tertiary health facility in Lagos. The specific objectives were: (1) to identify risk-factors for lethal arrhythmia of TdP; including prolonged corrected QT (QTc), and hypokalemia, (2) to determine the frequency of malaria infection, and (3) to identify types of anti-malaria agents (AMA) prescribed in HF.

\section{Methods}

Study location was the Lagos University Teaching Hospital (LUTH), Lagos; one of the two university-based tertiary health facilities in Lagos State, Nigeria. Ethical clearance for the study was obtained from the LUTH ethical committee.

\subsection{Design}

The study is a retrospective review of admitted HF patients; with a prospective evaluation of the ECG-QTc. With the help of the Records Department of the Institution, we obtained case-notes of all adult patients who were admitted into the medical wards with a diagnosis of HF, from January 1, 2010 to June 30, 2011. Our selection criteria were: adult patients aged $\geq 18$ years; availability of admission 12-lead ECG, admission blood biochemistry results, and echocardiography results. Case ascertainment for acute heart failure was the evidence of diagnosis made by unit senior registrar or consultant and evidence of management for $\mathrm{HF}^{[5]}$. The presence of HF was based on Framingham criteria ${ }^{[5,15]}$. Aetiology of underlying cardiac disease was based on clinical diagnosis as well as echocardiographic evidence. Majority of $\mathrm{HF}$ patients of our hospital have echocardiograms before discharge ${ }^{[5]}$. Exclusion criteria were: unavailability of requisite investigations, such as ECG, echocardiography or admission blood biochemistry reports. Other exclusion criteria were presence of clinical or laboratory data consistent with severe malaria, such as coma, severe anaemia ${ }^{[12,16]}$; and ECG features that disallow accurate QTc calculation, such as atrial fibrillation, left bundle branch block, and right bundle branch block ${ }^{[8,9]}$. 


\subsection{Malaria diagnosis}

(1) Evidence of plasmodium species parasitological diagnosis was by microscopy or rapid diagnostic tests ${ }^{[16]}$.

(2) Presumptive/suspected malaria diagnosis was based on prescription of anti-malaria agents ${ }^{[16,17]}$.

\subsection{Medication analysis}

World Health Organization (WHO) - designed proforma for rational drug use in chronic conditions was used. Use of this proforma requires a minimum of one hundred case-notes for useful data and valid conclusions (Source: World Health Organization Collaborating Centre for Drug Statistics Methodology).

We modified the proforma by excluding drug doses and duration of treatment. One of the investigators CAB - a postgraduate clinical pharmacist abstracted all data from the identified case-notes, using study inclusion and exclusion criteria. Study data abstracted included age, sex, aetiology of heart failure, number of cardiovascular drugs, treatment with AMA, and parasitological confirmation of malaria diagnosis. Selected risk-factors for TdP were advanced age, defined as $\geq 65$ years; bradycardia, defined as ECG heart rate $<60$ per minute; low serum potassium, defined as blood potassium $\leq 3.0 \mathrm{mmol} / \mathrm{L}$, ECG prolonged corrected QT interval (QTc) in males and females. Prolonged QTc was taken as $450 \mathrm{~ms}$ in men and $470 \mathrm{~ms}$ in women ${ }^{[18,19]}$.

The electrocardiogram (ECG) used for QTc calculation was a routine admission standard 12-lead simultaneous tracing and a lead II rhythm strip (at a paper speed of $25 \mathrm{~mm} / \mathrm{sec}$ and standard setting). A minimum of three cardiac cycles were recorded for each lead. One of us - CAB also prospectively calculated the QT interval and the corrected QT (QTc); using the manual eye-ball/calliper method ${ }^{[18,19]}$. The QT interval was measured from beginning of QRS complex to the end of the T-wave offset in leads II and V5, using three consecutive cardiac cycles, and their average values. The obtained QT interval was corrected for heart rate, by using the Bazett's formula, as follows: $\mathrm{QTc}=\mathrm{QT} /(\mathrm{RR}) 1 / 2^{[9,18,19]}$. The values were recorded in milliseconds.

\subsection{Study operative terms}

- "Advanced age" was defined as age $\geq 65$ years;

- Hypokalemia defined as serum potassium of $\leq 3 \mathrm{mmol} / \mathrm{L}$;

- Abnormal QTc: males- $\geq 450 \mathrm{~ms}$; women- $\geq 470 \mathrm{~ms}$;

- Bradycardia: heart rate $<60$ beats per minute;

- Group 1: HF patients with prescription of AMA;

- Group 2: HF patients without AMA;

- Artemisinin-based treatment: prescription with artemether, artesunate, dihydroartemisinin, paluther;

- Partner drugs: other antimalaria agents combined with artemisinin agents, e.g., sulphadoxine-pyrimethamine (SP), lumefantrine, piperaquine, amodiaquine, etc ${ }^{[16]}$.

\subsection{Statistical analysis}

Data obtained were documented in a proforma designed for the study and analysed using Epi-Info software version 3.51. Continuous parameters were recorded as means $\pm S D$. Descriptive data were given in numbers and percentages. Group comparison was based on presence and absence of anti-malaria treatment/prescription in groups 1 and 2, using Student's $T$-test or Chi-square analysis, where necessary. 


\section{Results}

The $160 \mathrm{HF}$ patients consisted of 82 males (51.3\%), 78 females $(48.7 \%)$, having an average ejection fraction of $39.6 \pm$ $12.6 \%$. HF aetiologies were predominantly non-ischemic. Table 1 summarised patients' general characteristics, showing that their mean age was $54.9 \pm 14.6$ years and the two chief underlying causes $(>80 \%)$ were hypertensive heart disease and dilated cardiomyopathy. Systolic blood pressure $>140 \mathrm{mmHg}$ was noted in 68 (43\%); while five was the median number of anti-HF and other cardiovascular medications. Figure 1 and Table 2 demonstrated that different individual risk factors of TdP occurred in 5\%-48\% of the patients; with hypokalemia and QTc prolongation occurring respectively in more than one-third of the total.

Table 1. General characteristics of the admitted acute heart failure patients

\begin{tabular}{ll}
\hline Parameters & Numbers (percentages); Means \pm SD \\
\hline Age (years) & $54.9 \pm 14.6$ \\
Gender: males (\%) & $82(51.7)$ \\
Heart rate beats/min & $96.0 \pm 19.6$ \\
SBP > $140 \mathrm{mmHg}(\%)$ & $68(42.5)$ \\
Aetiology: HHD/DCM/Others (\%) & $86(53.8) / 60(37.5) / 14(8.75)$ \\
Ejection fraction “EF” & $39.6 \pm 12.6$ \\
Preserved ejection fraction (\%) & $34(21.3)$ \\
Blood potassium (mmol/L) & $3.9 \pm 1.2$ \\
QTc ms & $469 \pm 79$ \\
Median length of hospital stay (days) & 16.5 \\
Malaria treated (\%) & $52(32.5)$ \\
Number CV medications & $4.74 \pm 1.0$
\end{tabular}

Note. $\mathrm{SBP}=$ systolic blood pressure; HHD = hypertensive heart disease; DCM = dilated cardiomyopathy; QTc = electrocardiograhic corrected QT interval.

Figure 1. Distribution of torsadogenic risk factors in the total study population

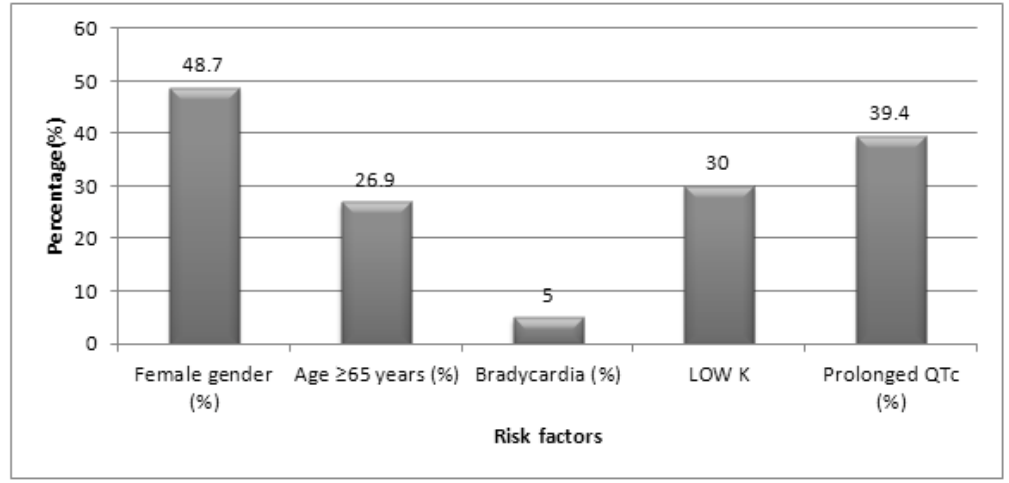

Table 2. Risk factors of torsades de pointes in the study population

\begin{tabular}{ll}
\hline Parameter (\%) & N (\%) \\
\hline Female gender (\%) & $78(48.7)$ \\
Age 65 years (\%) & $43(26.9)$ \\
Bradycardia (\%) & $8(5.0)$ \\
Blood potassium levels: LOW NORMAL - HIGH & $48(30 \%)-104(65 \%)-8(5 \%)$ \\
Prolonged QTc (\%) & $63(39.4)$ \\
\hline
\end{tabular}

Table 3 is a summary of the anti-malarial agents prescribed for these patients. Group 1 patients consisted of the 52 patients $(32.5 \%)$ with anti-malaria treatment; though only six patients $(11.5 \%)$ had parasitological confirmation. Importantly, all 
52 antimalaria-containing prescriptions were artemisinin-based; with 6 (11.5\%) of them as monotherapy. The artemisinincontaining agents, as prescribed were artesunate, artemether, and dihydroartemisinin. The anti-malaria partner drugs of the 46 artemisinin-combined treatment were SP in 27 (58.7\%), lumefantrine in $7(15.2 \%)$, amodiaquine in $7(15.2 \%)$ and piperaquine in $5(10.9 \%)$.

Table 3. Distribution of anti-malaria agents prescribed in the study population

\begin{tabular}{|c|c|c|}
\hline Drug type & Number & Percentage (\%) \\
\hline Artesunate + amodiaquine & 7 & 13.6 \\
\hline Artemether $20 \mathrm{mg}$ Lumefantrine 120 & 7 & 13.6 \\
\hline Artesunate + fansidar & 11 & 21.1 \\
\hline Artesunate only & 3 & 5.8 \\
\hline Alaxin only (dha $60 \mathrm{mg}$ ) & 3 & 5.8 \\
\hline p-alaxindha 40mg + ppq $320 \mathrm{mg}$ & 5 & 9.6 \\
\hline Cotexcin $($ dha $60 \mathrm{mg})+$ fansidar & 14 & 26.8 \\
\hline Paluther + fansidar & 2 & 3.7 \\
\hline Total & 52 & 100 \\
\hline
\end{tabular}

Note. Names of antimalaria agents as prescribed; fansidar $=$ SP (sulphodoxine-pyrimethamine; dha $=$ dihydroartemisinin; $p$ pq $=$ piperaquine

Table 4 summarised the comparison of TdP factors in 52 (Group 1) and 108 (Group 2) patients; showing that mean age, heart rate, QTc and serum potassium were comparable $(\mathrm{p}>.05)$. However, using categorisation in Table 4 showed that the proportion of older patients ( $\geq 65$ years), prolonged QTc and serum potassium differed significantly between the two groups. Table 5 and Figure 2 show demonstrate the significant association of serum potassium (K) status with AMA treatment groups.

Table 4. Comparison of selected risk factors of torsades de pointes among HF patients

\begin{tabular}{lll}
\hline Parameter & Group I $(\mathbf{n}=\mathbf{5 2})$ & Group II $(\mathbf{n}=\mathbf{1 0 8})$ \\
\hline Age years & $57.1 \pm 14.6$ & $52.5 \pm 14.3 ; p=.15$ \\
Age $>$ 65 years (\%) & $20(38.5)$ & $23(21.3) ; p=.02^{*}$ \\
Heart rate per minute & $89.5 \pm 21.0$ & $92.8 \pm 19.0 ; p=.57$ \\
Bradycardia (\%) & $3(5.8)$ & $5(6.0) ; p>.05$ \\
Serum potassium mmol/L & $3.74 \pm 0.68$ & $3.83 \pm 0.64 ; p=.6$ \\
ECG QTc ms & $458.7 \pm 55.3$ & $468.0 \pm 61.8 ; p=.54$ \\
Prolonged QTc (\%) & $25(48.1)$ & $38(35.2) ; p=.1^{*}$ \\
Females & $25(48.1)$ & $53(49.1) ; p>.05$ \\
Length of hospital stay days & $16.0 \pm 9.3$ & $16.6 \pm 9.5 ; p>.05$ \\
\hline
\end{tabular}

Note. $\mathrm{QTc}=$ corrected QT interval; group $1=$ antimalaria agents; ${ }^{*}=$ significant association

Figure 2. Distribution of serum potassium in patients treated with and without anti-malaria agents

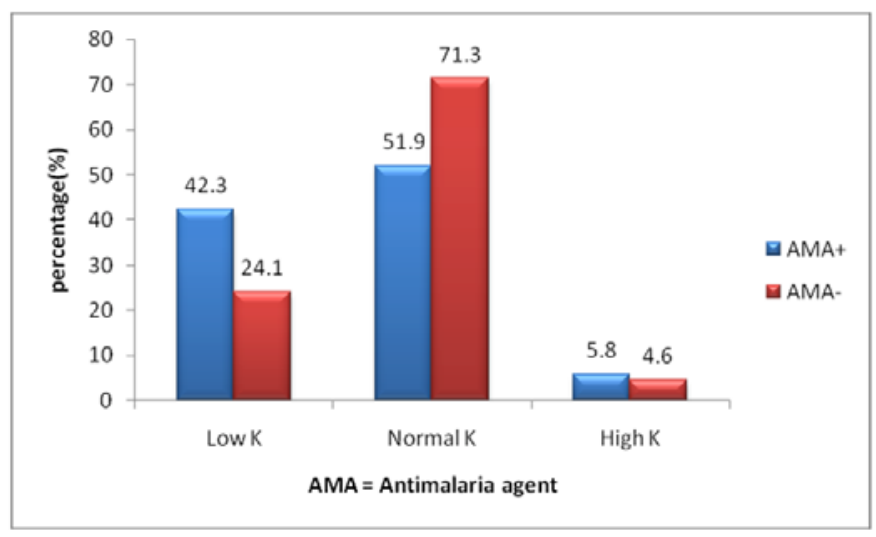


Table 5. Distribution of serum potassium $(\mathrm{K})$ in patients with and without anti-malaria agents

\begin{tabular}{lllll}
\hline & Low $\mathbf{K}$ & Normal K & High $\mathbf{~}$ & Total \\
\hline Group 1 AMA+ & 22 & 27 & 3 & 52 \\
GROUP 2 AMA- & 26 & 77 & 5 & 108 \\
Total & 48 & 104 & 8 & 160 \\
\hline
\end{tabular}

Note. $\mathrm{X}^{2}=6.01, p=05 ; \mathrm{K}=$ serum potassium status

\section{Discussion}

This study investigated the pattern of malaria treatment and frequency of predisposing risk factors of torsades de pointes among the admitted HF population. The study also examined whether these risk factors differed significantly among the malaria-treated group. Our patients' general characteristics are comparable to those of patients admitted in similar centres in Nigeria and Africa ${ }^{[3-5,8,9]}$; especially regarding non-ischemic aetiology, mean age in the mid-50s and degree of left ventricular dysfunction ${ }^{[3-5,8,9]}$. Our patients, however differed from those of the industrialised world, where HF occurs mainly in the elderly, and the predominant aetiology is ischemic ${ }^{[1,2,6]}$. In the present study, only a quarter of our patients were older than 65 years.

In the present study also, it is note-worthy that the frequency of individual risk factors for $\operatorname{TdP}^{[7,13,14]}$ were variable, ranging from 5\%-48\%. Approximately $40 \%$ of our HF patients exhibited prolonged QTc, which was lower than that found in other studies from other States of Nigeria ${ }^{[8,9]}$. However, different studies have used different cut-off values for prolonged QTc ${ }^{[7-10,18,19]}$. For instance, prolonged QTc was seen in $60 \%$ of HF patients from the Northern part of Nigeria using cut-off values of $420 \mathrm{~ms}$ for men and $440 \mathrm{~ms}$ for women ${ }^{[9]}$. It is however important that the mean QTc values of the different HF studies were similar. The mean QTe values were also significantly different from non-affected controls ${ }^{[8,9]}$.

Several reviews and authors have emphasised that TdP risk factors also include underlying cardiac disease, especially HF and dilated cardiomyopathy ${ }^{[7,13,14]}$. These conditions already exist in our patients; thus placing them intrinsically at higher cardiac risk. Our data indicated that one third of the hospitalised HF patients received prescriptions for malaria treatment. This proportion is high and these patients would carry a higher disease and pill burden ${ }^{[17]}$. The fact that the malaria diagnoses were predominantly presumptive ${ }^{[16,17]}$ is also note-worthy. It is rather surprising that even at this level of health care, the parasitological confirmation was minimal. However, it is pertinent to note that at the time of the study, the National policy on malaria treatment was not so anchored on parasite confirmation ${ }^{[16]}$. Such malaria-treated HF patients were clearly exposed to a further increase in the total number of medications; with resultant higher poly-pharmacy. This clinical scenario would contribute to cardiovascular and pharmacologic adverse effects ${ }^{[7,10,14]}$.

Our interest was to investigate if malaria-treated patients possessed a higher frequency of torsadogenic risk factors compared to those without malaria treatment. Thus, we compared the pre-treatment risk profile of anti-malaria group with that of non-malaria treated group. Interestingly, mean values of studied risk factors were comparable in the two groups; but using univariate analyses indicated that affected proportions; including those with hypokalemia and prolonged QTc differed significantly in the two groups. Since these evaluations were pre-malaria treatment, it would suggest that the noted differences would be a reflection of pre-existing factors, such as a malaria effect. There is evidence that malaria on its own significantly affected the cardiovascular system, with effects of morphological alterations, such as conduction defects on the ECG, and blood pressure changes of hypotention and elevated blood pressure ${ }^{[22,23,26]}$. Some authors attributed such findings to possible release of pyrogenes, cytokine-related effects, including vasodilatation ${ }^{[22-24]}$. Our opinion is that these findings should be a stimulus for further insight into possible effects of malaria in heart failure patients.

We also investigated the choice of AMA used in this clinical scenario; as certain AMA has marked cardiovascular adverse effects ${ }^{[10-14]}$. We opined that prescribers at this level of health care would recognize that the status of cardiac dysfunction 
would contribute to a high potential for toxicity. Thus our expectation was that their antimalaria choices would reflect appreciable cardio-safety. The anti-malaria agents prescribed in this study were $100 \%$ artemisinin-based and there were combinations with other malaria partner-drugs in $90 \%$. The WHO and National Malaria Policy currently recommend artemesinin-based combinations ${ }^{[16]}$. In our study, the choice of malaria partner-drugs was predominantly SP and different 4-aminoquinolines, namely lumefantrine, amodiaquine, and piperaquine. To date, artemisinin derivatives enjoy a favourable cardiovascular profile as reported in normal individuals with malaria, in experimental situations, and in usual doses ${ }^{[10,16,21,25]}$. However even such reports have acknowledged electrical abnormalities, including QRS morphological alterations, and blood pressure changes, though with unclear clinical implications ${ }^{[16,21,25]}$.

With regard to partner-drugs, SP has enjoyed a long experience; but reports noted that adverse effects were uncommon and affected other systems rather than the cardiovascular system ${ }^{[20]}$. The 4-aminoquinolines are important partnerdrugs to artemisinin; and those with important cardiovascular issues, including QTc prolongation are quinine, quinidine, halofantrine and possibly mefloquine ${ }^{[7,10,16]}$. However these drugs were not used in this cohort. Lumefantrine, amodiaquine and piperaquine as partner-drugs appear to have a safe cardiovascular profile ${ }^{[11,16,20]}$. However, recent publications are calling for caution with these drug classes, as changes in antioxidant status and adverse renal changes have been observed ${ }^{[25]}$. The authors noted that piperaquine is a bisquinoline and structurally related to chloroquine. Thus it was expected to exhibit electrophysiological effects on the heart ${ }^{[25]}$. We also reiterate that caution be exercised on the use of lumefantrine and piperaquine combinations in cardiac patients because of their close structural resemblance to halofantrine and other 4-aminoquinolines. Some investigators have cautioned that clinical drug evaluations and drug toxicity tests be carried out using single agents and on non-cardiac, and previously healthy malaria patients ${ }^{[27]}$. Thus, in a real life situation of admitted patients, certain cardiac issues may not be easily predictable ${ }^{[27]}$. It is known that multiplicity of medications and complex drug regimens increase tendency of adverse drug-drug interactions ${ }^{[7,10,14,27]}$, which could sometimes be caused by drug metabolites ${ }^{[28]}$ or drugs with potential to disturb important drug-elimination mechanisms ${ }^{[2]}$. Thus further studies are required especially on the use of 4-amino-quinololines such as lumefantrine and piperaquine in heart failure patients. Drug reviews have previously noted that amodiaquine as a single agent was shown to increase heart rate and resulted in toxic effects on some liver and renal function tests ${ }^{[20,25]}$. The authors cautioned that although certain medications which did not have a direct effect on the heart; but altered hepatic and renal profiles may indirectly affect the heart ${ }^{[20,25]}$ by affecting drug metabolism of heart failure drugs or malaria drugs ${ }^{[25]}$.

Along these lines, and attempting to examine the effect of artemisin-combination on the heart, some investigators in Ghana examined QTc interval prologation on children aged 4-12 years, with uncomplicated malaria ${ }^{[30]}$. They found significantly longer QTc on day 0 compared to QTe values on day 3 and day 7. The authors opined that the longer QTc of untreated malaria phase suggested a disease-specific effect of malaria. However there was $>30$ seconds increase in baseline QTc in $31.9 \%$ of patients on artemisinin-amodiaquine versus $20 \%$ on artemisinin-lumefantrine combination. The authors therefore called for continuing caution and further studies in the possible QTc effects of malaria and the artemisinin combinations.

\section{Strengths and limitations of the study}

The study was a retrospective one, and some limitations of retrospective study include missing data. But this limitation was minimised by the inclusion and exclusion criteria for the selected cases, with all the important factors for the study available. It is important to note that retrospective studies are important since such studies help in the investigation of real-life situations and clinical practice ${ }^{[5]}$. A notable strength of our study was the removal of selection bias by using a study cohort that was all-inclusive. Our most important inclusion criterion was the availability of admission ECG on adults admitted with HF. Since our Hospital protocol demanded that HF patients have ECG within the first twenty-four hours of admission ${ }^{[5]}$, data from all adult HF patients were likely to be "captured". In addition, the involvement of Records Department of our Hospital assured more complete "catchment" of the HF patients admitted during the study period. Although the sample size may appear small, but the drug data-collection instrument of World Health Organization used in 
this study requires a minimum of one hundred case-records for valid conclusions (see methodology). Thus our study cohort of one hundred and sixty appears adequate.

Our study was also unique in that we prospectively measured the ECG-QTc, in order to take all the necessary precautions ${ }^{[18,19]}$. The ECG-QTc has been adjudged a valuable method and described as standard of clinical care on issues of cardio-toxicity ${ }^{[10,14]}$. This is because of its simplicity, availability, and wide use in the clinical scenario. Thus results can be easily compared to other studies. Touze et al. ${ }^{[10]}$ emphatically recommended that pre-treatment QTc values must be used when quinine, halofantrine and others are to be prescribed in clinical situations that further increase cardiac toxicity, such as use of diuretics, laxatives, selective H1 receptor antagonists. Such medications modify or block the delayed rectifier K+ channels, resulting in prolonged QTc - a harbinger of TdP ventricular tachycardia. The present study was thus carried out to examine the pre-treatment cardiac risk profile of the HF patients with malaria. Touze and his colleagues also argued that such pre-treatment evaluation would result in the correction of any electrolyte imbalance ${ }^{[10]}$.

\section{Conclusions}

Our study showed that one third of admitted HF patients received a diagnosis of malaria. Using malaria pre-treatment evaluation, we identified several different risk factors of torsades de pointes amongst the HF patients even prior to malaria treatment. Interestingly, the risk factor profile of the malaria-treated HF patients also appeared significantly worse than the group without anti-malaria treatment, inviting the possibility of additional "malaria" effect. All malaria treatment was artemisinin-based but the anti-malaria partner drugs were mainly SP and 4-aminoquinolines. Given the frequency of malaria and HF in high transmission communities, and the high risk of their occurrence, our findings should contribute in future to safer malaria treatment practices, and selection of safer drugs in admitted HF patients with malaria. In the present study, malaria pre-treatment evaluation had uncovered the frequency of increased cardiotoxic potential in both malaria and non-malaria HF patients. Our study further high-lights the heighted cardiotoxic risk profile of HF in high transmission malarious areas.

\section{Competing interests}

The authors declare that they have no competing interests.

\section{Acknowledgements}

We appreciate the staff of Records Department in LUTH for their help in theprovision of case-notes.

\section{References}

[1] Hunt SA, Abraham WT, Chin, MH, et al. Focused update incorporated into ACC/AHA 2005 Guidelines for the diagnosis and management of heart failure in the adults: a report of American College of Cardiology Foundation / American Heart Association Taskforce on practice guidelines: developed in collaboration with International Society of Heart and Lung transplantation. Circulation. 2009; 119: e391-e479. PMid: 19324966. http://dx.doi.org/10.1161/CIRCULATIONAHA.109.192065

[2] McMurray JJV, Adamopoulos S, Anker SD, Auricchio A, Bohm M, Dickstein K, et al. The Task Force for Diagnosis and Treatment of Acute and Chronic Heart Failure 2012 of the European Society of Cardiology. Eur Heart J. 104: 1-61. http://dx.doi.org/10.1093/eurheartj/ehs

[3] Adebayo KA, Adebiyi AA, Oladapo OO, Ogah OS, Aje A, Ojji DB, et al. Characterisation of heart failure with normal ejection fraction in a tertiary hospital in Nigeria. BMC Cardiovasc Dis. 2009; 9: 52. http://dx.doi.org/10.1186/1471-2261-9-52

[4] Damesceno A, Mayosi BA, Sani M, Ogah OS, et al. The causes, treatment and outcome of acute heart failure in 1006 Africans from 9 countries. Arch of Intern Med. 2012. http://dx.doi.org/10.1001/archinternmed.2012.3301

[5] Ajuluchukwu JN, Anyika EN, Raji KA. Physician adherence to pharmacotherapy guidelines for chronic heart failure in a tertiary health facility in Lagos-Nigeria. Journal of Hosp Administration. 2014; 3 (2): 32-41. Available from: www.sciedu.ca/jha

[6] Ruduger A, Harjola V, Andras M, Mattla E, Saila P, Niemuren M, Follath F. Acute heart failure: clinical presentation, one year mortality and prognostic factors. Europ J Heart Fail. 2005; 7: 660-670. 
[7] Roden DM. Drug induced prolongation of QT interval. New Engl J Med. 2004; 350: 1013-1022. PMid: 14999113. http://dx.doi.org/10.1056/NEJMra032426

[8] Opadijo OG. Effect of lisinopril on QTc and arrhythmias in patients with congestive heart failure. Nig JInt Med. $2000 ; 3: 50-53$.

[9] Kolo PM, Opadijo OG, Omotosho ABO, Balogun MO, Araoye MA, Katibi JA. Prevalence of QTcprolongation in adult Nigerians with chronic heart failure. West Afr J Med. 2008; 27(2): 69-73. PMid:19025017.

[10] Touze JE, Heno P, Forcade L, Deharo JC, Thomas G, Bohan S, et al. The effects of anti-malaria drugs on ventricular repolarisation. Am J Trop Med Hyg. 2002; 67: 54-60. PMid:12363064.

[11] Coker S, Batey AJ, Lightbown IA, Diaz ME, Eisner DA. Effects of mefloquine on cardiac contractility and electrical characteristics in-vivo, in isolated cardiac preparations, and in single ventricular myocytes. British J Pharm. 2000; 129: 323-330. PMid: 10694239. http://dx.doi.org/10.1038/sj.bjp.0703060

[12] Hall AP: The treatment of malaria. British Med J. 1976; 1: 323-327. PMid: 764937. http://dx.doi.org/10.1136/bmj.1.6005.323

[13] Bouchad O, Impert P, Touze JE, Dodoo AN, Danis M, Legros F. Fatal cardiotoxicity of halofantrine: A review based on a world-wide safety data base. Malar J. 2009; 8: 289-292. PMid: 20003315. http://dx.doi.org/10.1186/1475-2875-8-289

[14] Yap YG, Camm AJ. Drug induced QT prolongation. Heart. 2003; 89: 1363-1372. http://dx.doi.org/10.1136/heart.89.11.1363

[15] Vasan RS, Benjamin EJ, Larson MG, Leip EP, Wang TJ, Wilson PWF, et al. Plasma natriuretic peptides for community screening for left ventricular hypertrophy and systolic function. The Framingham Study. JAMA. 2002; 288: 1252-59. PMid: 12215132. http://dx.doi.org/10.1001/jama.288.10.1252

[16] National policy on malaria Federal Republic of Nigeria. Nigeria guidelines for diagnosis and treatment of malaria. 2011.

[17] Odusanya OO. The role of adjunct therapies in the rational prescription of uncomplicated malaria. Nig J Clin Practice. 2002; 5: 32-36.

[18] Goldenberg I, Moss AJ, Zareba W. QT interval: how to measure it and what is normal. Journal of Cardiovasc Electrophysiology. 2006; 17: 333-336. PMid: 16643414. http://dx.doi.org/10.1111/j.1540-8167.2006.00408.x

[19] Ale OK, Ajuluchukwu JNA, Oke DA, Mbakwem AC. QT dispersion in hypertensive Nigerians with and without left ventricular hypertrophy. WAMJ. 2013; 32 (1): 57-61.

[20] Phillips-Howard PA, West LJ. Serious adverse drug reactions to pyrimethaminesulphadoxine, pyrimethamine-dapsone and amodiaquine in Britain. J Royal Soc Med. 1990; 83: 82-84. PMid: 2138674.

[21] Hien TT, White NJ. Qinghaosu. The Lancet. 1993; 341: 603-607. http://dx.doi.org/10.1016/0140-6736(93)90362-K

[22] Anigbogu CN, Lawal TA. Effects of malaria on blood pressure, heart rate, and ECG in man. NigQt J Hosp Med. 2000 ; 10 : 63-68.

[23] Zaki SA, Shanbog P. Atypical manifestations of malaria. Res \& Reports Trop Dis. 2011; 2: 9-22. http://dx.doi.org/10.2147/RRTM.S13431

[24] White NJ. Cardiotoxicity of antimalaria drugs. Lancet Infect Dis. 2007; 7: 549-58. http://dx.doi.org/10.1016/S1473-3099(07)70187-1

[25] Olayinka ET, Ore A. Alterations in anti-oxidant status of and biochemical indices following administration of dihydroartemisinin and piperaquine combination (P-alaxin) IOSR J Pharm and Biol Sciences. 2013; 5: 43-53. http://dx.doi.org/10.9790/3008-0544353

[26] Onwuamaegbu ME, Henein M, Coats AJ. Cachexia in malaria and heart failure: Therapeutic Considerations in Clinical Practice. Postgrad Med J. 2004; 80: 642-649. PMid: 15537847. http://dx.doi.org/10.1136/pgmj.2004.020891

[27] Mirams GR, Cui Y, Sher A, Fink M, Cooper J, Heath BM, et al. Simulation of multiple ion channel block provides improved early prediction of compound's clinical torsadogenic risk. Cardiovasc Res. 2011; 91: 53-61. PMid: 21300721. http://dx.doi.org/10.1093/cvr/cvr044

[28] Abernethy DR, Flockhart DA. Molecular basis of cardiovascular drug mechanism: implications for predicting clinically important drug interactions. Circulation. 2000; 101: 1749-53. http://dx.doi.org/10.1161/01.CIR.101.14.1749

[29] Hochman S, Kim K. The impact of HIV coinfection on cerebral malaria pathogenesis. Journal of Neuroparasitology. $2012 ; 3$. http://dx.doi.org/4303/jnp/235547.

[30] Adjei GO, Odiro-Boateng C, Rodrigues OP, Hoegberg LC, Alifrangis M, Goka B. Electrocardiographic study in Ghanaian children with uncomplicated malaria treated with artesunate-amodiaquine or artemether-lumefantrine. Malaria Journal. 2012; 11: 420-427. PMid: 23244570. http://dx.doi.org/10.1186/1475-2875-11-420 